\title{
Grain Boundary Studies in CuInGaSe 2 Using EBSD in the Dual Beam FIB
}

\author{
Brian P. Gorman and Michael J. Kaufman \\ Department of Materials Science and Engineering, University of North Texas, Denton, TX, USA \\ 76203
}

Matt M. Nowell

TSL / EDAX, Inc., Draper, UT, USA

Seokhyun Yoon and Tim Anderson

Department of Chemical Engineering, University of Florida, Gainesville, FL, USA

Chalcopyrite structured II-IV-V $\mathrm{V}_{2}$ and I-III-VI $\mathrm{V}_{2}$ semiconductors are important materials for polycrystalline thin film photovoltaics. Specifically, CuInSe ${ }_{2}$ and $\mathrm{CuInGaSe}_{2}$ (CIS and CIGS) have a high tolerance for defects and stoichiometry changes. Unlike other optoelectronic semiconductors, CIS and CIGS have shown stable polar $<112>$ facets. This results in $\mathrm{Cu}$ vacancies or interstitials, which has been suggested [1] to be responsible for the enhanced grain boundary conductivity. These facets have been observed experimentally using AFM and XRD [2], but grain boundary normals (defining the structure of the grain boundaries) have not been examined on the localized scale. The ability to do so may give insight into the origin of their formation during growth, and ultimately lead to higher conductivity materials.

In this study, EBSD was used to study the frequency of formation of these $<112>$ grain boundaries. $2 \mu \mathrm{m}$ thick CIGS samples were grown using CVD on Mo-coated glass substrates. Uncoated samples were imaged in an FESEM at $20 \mathrm{keV}$ in high-resolution mode $(5 \mathrm{~mm}$ working distance and in-lens detector, figure 1). An attached EBSD system (EDAX / TSL, Inc.) was used to map the grain normals of these samples, from which maps of the grain boundary trace normals could be determined. A pole figure about the (111) axis is shown in figure 2. An inverse pole figure and texture map is illustrated in figure 3. As can be seen, the samples are preferentially textured about this axis.

In order to determine the grain boundary normals (3-D vs. 2-D grain boundary traces), EBSD experiments were conducted in an FEI Nova 200 Nanolab dual beam FESEM / FIB. Initially rough surfaces were milled using a $5 \mathrm{keV}$ accelerating voltage on the ion column (figure 4), and subsequent mills allowed for 3-D examinations of the grain boundary trace normals. The limits of this grain boundary normal analysis technique will be discussed in detail. Once $<112>$ grain boundary trace normals are determined, TEM samples can be made normal to the grain boundaries and directly examined in the TEM. High resolution TEM imaging can confirm the presence of $<112>$ boundaries, and quantitative STEM EELS spectroscopy can confirm changes in Cu stoichiometry at the boundaries.

\section{References}

[1] John E. Jaffe and Alex Zunger, J. Phys. And Chem. Of Solids, 64 (2003) 1547.

[2] D. Liao and A. Rockett, J. Appl. Phys., 91 (2002) 1978.

[3] T. L. Matteson, S. W. Schwartz, E. C. Houge, B. W. Kempshall, L. A. Giannuzzi, J. Electron. Mat., 31 (2001) 33. 


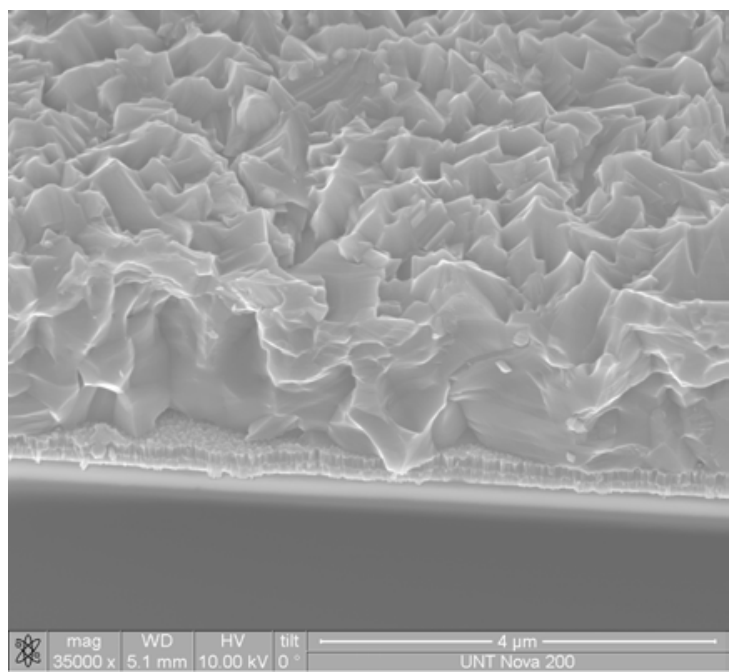

FIG. 1. FESEM image of as-grown CIGS thin film

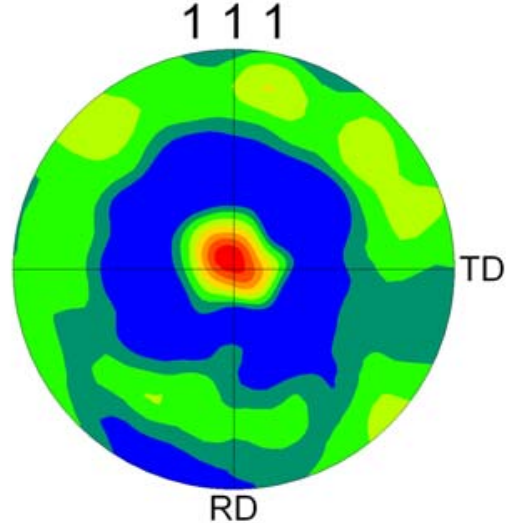

FIG 2. $<111>$ texture map of as-grown CIGS
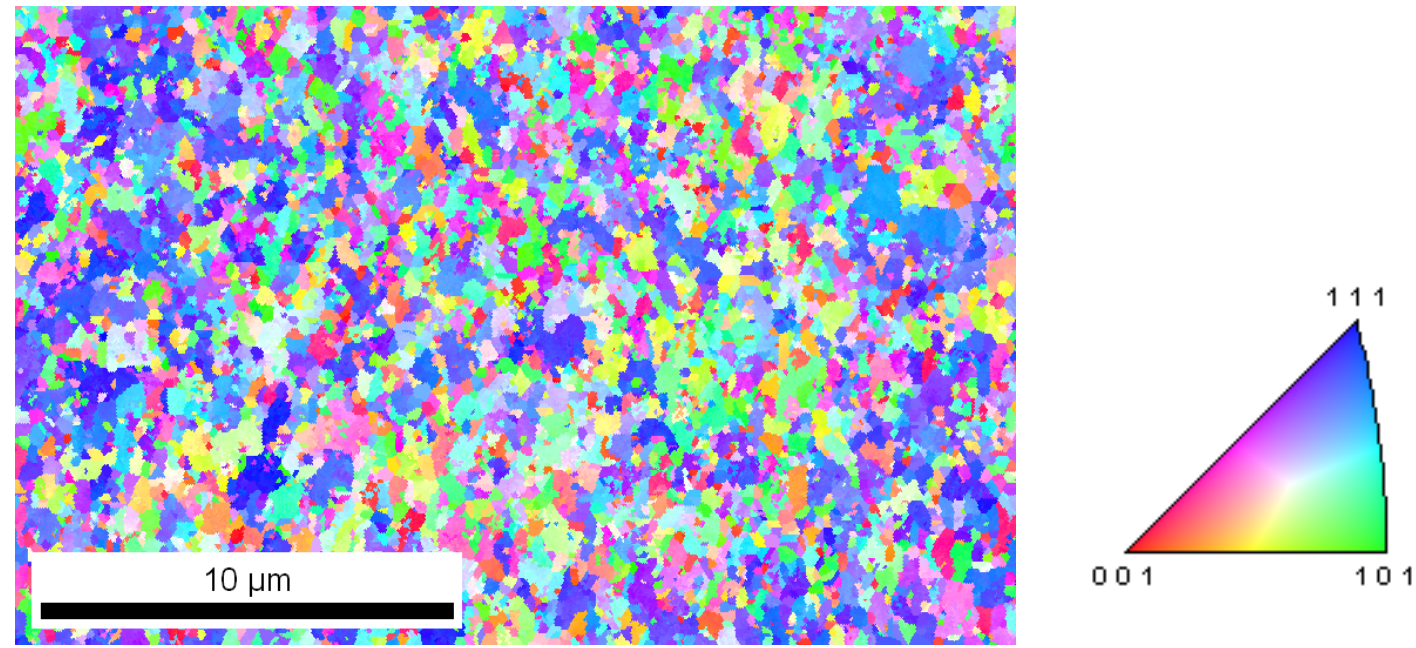

FIG 3. Inverse Pole Figure of as-grown CIGS.
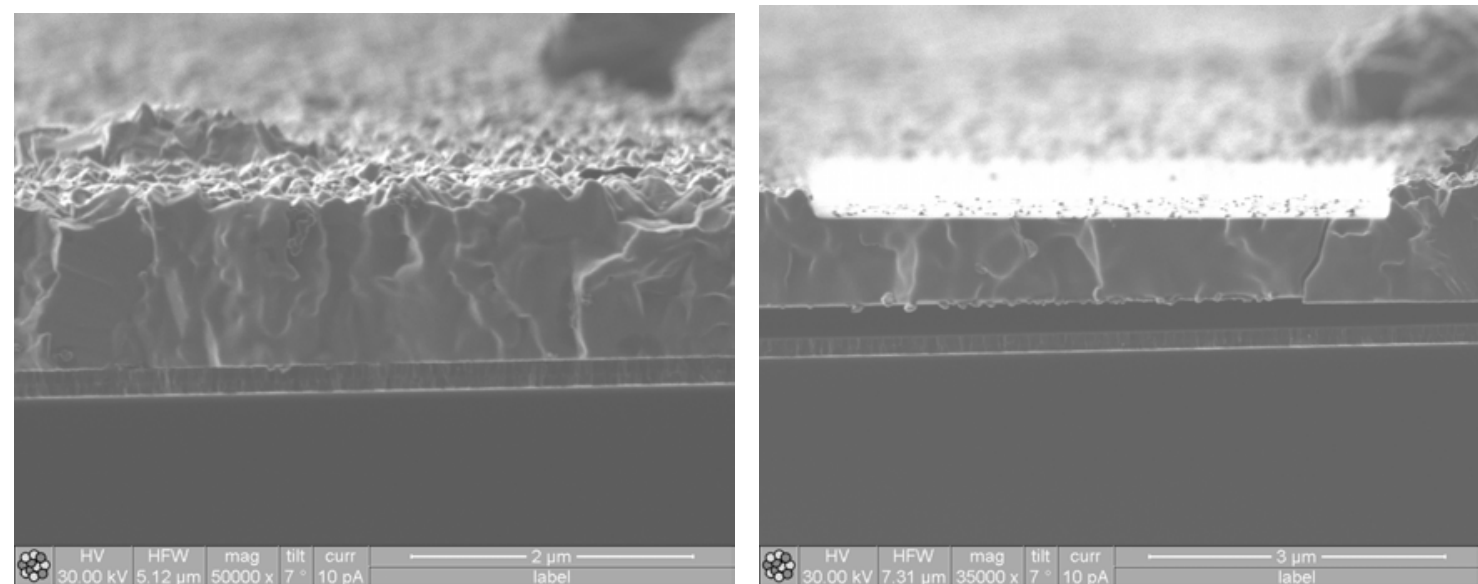

FIG 4. Ion image of CIGS cross section after surface milling. The necessary resolution for 3-D reconstructions of grain boundaries in CIGS is illustrated. 